\title{
The Activity of Pax3 and Zic1 Regulates Three Distinct Cell Fates at the Neural Plate Border
}

\author{
Chang-Soo Hong and Jean-Pierre Saint-Jeannet
}

\author{
Department of Animal Biology, School of Veterinary Medicine, University of Pennsylvania, \\ Philadelphia, PA 19104
}

Submitted November 28, 2006; Revised February 7, 2007; Accepted March 27, 2007

Monitoring Editor: Marianne Bronner-Fraser

\begin{abstract}
In Xenopus, the neural plate border gives rise to at least three cell populations: the neural crest, the preplacodal ectoderm, and the hatching gland. To understand the molecular mechanisms that regulate the formation of these lineages, we have analyzed the role of two transcription factors, Pax3 and Zic1, which are among the earliest genes activated in response to neural plate border-inducing signals. At the end of gastrulation, Pax3 and Zic1 are coexpressed in the neural crest forming region. In addition, Pax3 is expressed in progenitors of the hatching gland, and Zic1 is detected in the preplacodal ectoderm. Using gain of function and knockdown approaches in whole embryos and animal explants, we demonstrate that Pax3 and Zic1 are necessary and sufficient to promote hatching gland and preplacodal fates, respectively, whereas their combined activity is essential to specify the neural crest. Moreover, we show that by manipulating the levels of Pax3 and Zic1 it is possible to shift fates among these cells. These findings provide novel information on the mechanisms regulating cell fate decisions at the neural plate border.
\end{abstract}

\section{INTRODUCTION}

At the end of gastrulation, the ectoderm of the vertebrate embryo can be divided into three major domains: the nonneural ectoderm and the neural plate separated by an intervening region known as the neural plate border (NPB). The nonneural ectoderm and neural plate will develop into epidermis and CNS, respectively. In Xenopus the NPB contains at least three cell populations: the neural crest (NC), the preplacodal ectoderm (PE), and the hatching gland (HG). The NC is located lateral to the neural plate but excluded from its most anterior region. The $\mathrm{PE}$ is restricted to the anterior region of the embryo, lateral to the neural crest, and at the most rostral boundary of the neural plate. The HG is confined to the superficial layer of the ectoderm of the anterior neural folds, medial to the NC (Figure 1, A and B).

Each one of these cell populations contributes a number of derivatives with important specialized functions in the developing embryo. Upon migration into the periphery, NC cells differentiate into a large variety of cell types, including peripheral neurons and glia, smooth muscle cells, craniofacial cartilage and bone, and endocrine and pigment cells (Gammill and Bronner-Fraser, 2003; Le Douarin et al., 2004). The PE eventually subdivides into individual placodes, which give rise to the paired sensory organs, and it also makes an important contribution to the peripheral nervous system (Streit, 2004; Brugmann and Moody, 2005; Schlosser, 2005). The HG produces proteolytic enzymes, which partially digest the vitelline envelope and jelly coat to release

This article was published online ahead of print in $M B C$ in Press (http:/ / www.molbiolcell.org/cgi/doi/10.1091/mbc.E06-11-1047) on April 4, 2007.

Address correspondence to: Jean-Pierre Saint-Jeannet (saintj@vet.upenn. edu).

Abbreviations used: HG, hatching gland; NC, neural crest; NPB, neural plate border; PE, preplacodal ectoderm. the tadpole into the environment (Drysdale and Elinson, 1991).

Evidence obtained primarily in Xenopus strongly suggests that two independent signals are necessary for NC induction: a Bmp signal, which must be attenuated by Bmp antagonists, and a separate signal mediated either by a canonical Wnt or Fgf (for reviews, see Knecht and Bronner-Fraser, 2002; Huang and Saint-Jeannet, 2004). It is thought that the same combination of signals (Bmp and Wnt) induces the HG concomitantly to the NC (McGrew et al., 1999). Some of the signals involved in the generation of the NC and the HG have also been implicated in the induction of the PE. In Xenopus, a balance of Bmp and its antagonists is in part responsible for positioning the PE (Brugmann et al., 2004; Glavic et al., 2004), and a recent study indicates that Fgf8 positively regulates the expression of the preplacodal-specific gene Six1 when Bmp is inhibited in the ectoderm (Ahrens and Schlosser, 2005). Interestingly, the PE seems to have different requirement with regard to $W n t$ signals compared with the $\mathrm{NC}$, because inhibition of the Wnt signaling pathway favors placodal tissue at the expense of NC fate (Brugmann et al., 2004). How these signals are integrated at the NPB to generate distinct fates is an important and still unresolved question.

Recently, Meulemans and Bronner-Fraser (2004) presented a comprehensive analysis of the regulatory network of genes expressed at the NPB, in which they proposed that in response to signaling events mediated by Bmp, Wnt, and Fgf, a number of transcriptions factors are sequentially induced at the NPB in a two-step process. First, a group of genes is activated, referred as "NPB specifiers," which includes members of the Zic, Pax, Dlx, and Msx families of transcriptional regulators. These factors, which are broadly expressed at the NPB, are in turn responsible for the activation of a subset of genes with more restricted expression domains, known as "NC specifiers" (Meulemans and Bronner-Fraser, 2004) or "PE specifiers" (Litsiou et al., 2005). The function of these NC and PE specifiers is to control the expression of genes 
A

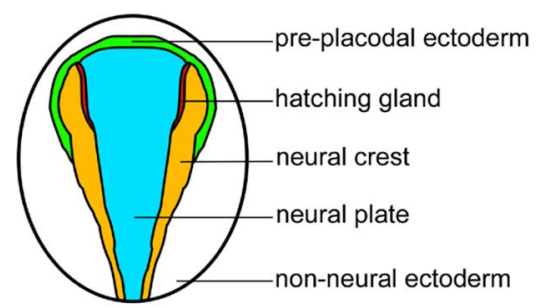

Figure 1. Molecular characterization of neural plate and NPB cells. (A) Dorsal view (anterior to top) of a schematic representation of an early neurula stage Xenopus embryo highlights the position of the neural plate, nonneural ectoderm, and intervening NPB cells that include the prospective $\mathrm{HG}, \mathrm{NC}$, and PE. (B) Schematic representation of a transverse section through a neurula stage embryo in which the different components of the ectoderm are color coded according to A. en, endoderm; so, somite; no, notochord. (C) Expression of neural plate- (Sox2) and NPB (Snail2, Xhe, and Six1)-specific genes by whole-mount in situ hybridization. Dorsal views, anterior to top. (D) Double in situ hybridization showing the relationship of Snail2 (NC; arrowhead) and Xhe (HG; arrow) expression domains. Lateral view, anterior to right. (E) Transverse section through neurula stage embryos illustrating the relative position of the presumptive HG (Xhe; arrows), NC (Snail2; arrowheads), and PE (Six1; bracket). (F) Schematic representation of cross-sections through neurula stage embryo showing the domain of expression of the Sox2, Xhe, Snail2, and Six1 (purple).
C

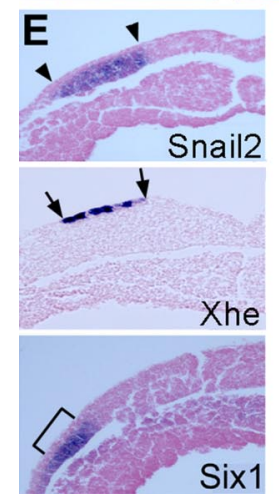

B

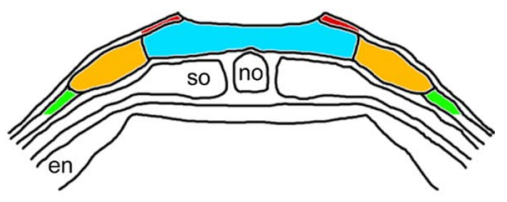

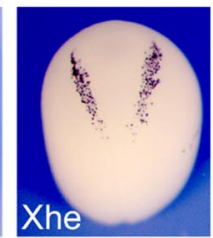

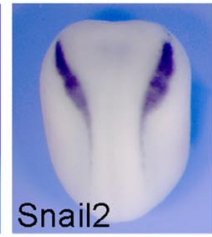

$\mathbf{F}$

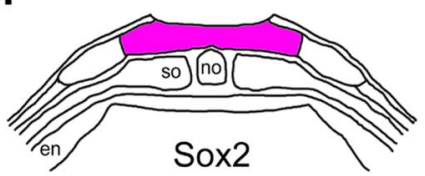

Sox2
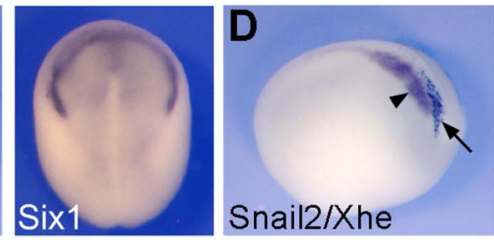

Snail2/Xhe

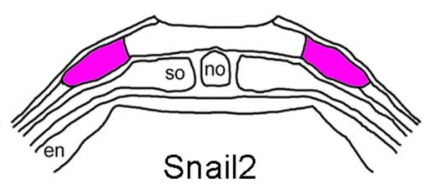

Snail2
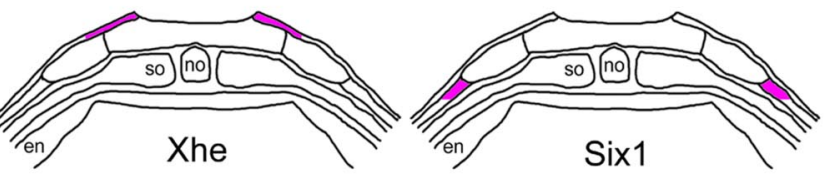

regulating the behavior and differentiation patterns of these two cell lineages.

To better understand the processes leading to the segregation of these populations of cells at the NPB, we have analyzed the function of two transcriptional regulators, Pax3 and Zic1. Both factors are broadly expressed at the NPB in response to Bmp, Wnt, and Fgf signaling. Although their expression domains overlap in the NC forming region, Pax3 and Zic1 are also expressed in the presumptive HG and PE, respectively. Here, we show that the combinatorial activity of these two transcription factors work to subdivide the NPB into three distinct cell fates.

\section{MATERIALS AND METHODS}

\section{Plasmid Constructions}

Pax3 (Monsoro-Burq et al., 2005) and Zic1 (Sato et al., 2005) constructs were generated by polymerase chain reaction (PCR) and fused in frame to the human glucocorticoid receptor (GR) ligand binding domain (Kolm and Sive, 1995). These fusion constructs subcloned into pCS2+, referred as Pax3GR and Zic1GR, respectively, were sequenced, and the corresponding protein was monitored using an in vitro transcription/translation coupled rabbit reticulocyte lysate system (Promega, Madison, WI).

\section{Xenopus Embryo Injections, Explants Culture, and Dexamethasone Treatment}

Embryos were staged according to Nieuwkoop and Faber (1967). Wnt1 (0.1 ng; Wolda et al., 1993), noggin (0.1-2 ng; Smith and Harland, 1992), Fgf8a (0.1-50 pg; Christen and Slack, 1997), Pax3GR (0.025-1 ng), and Zic1GR (0.025-1 ng) mRNAs were synthesized in vitro by using the Message Machine kit (Ambion, Austin TX). Pax3 (Pax3MO; TCTCAGTTCCCTTGCCAAGTATTAA; Monsoro-Burq et al., 2005), Zic1 (Zic1MO; AAGTCTTCCAACAATGGGCAGCGAA; Sato et al., 2005), and Fgf8a (Fgf8aMO; CTCTGCTCCCTCACATGCTGTGTAA; Fletcher $e t$ al., 2006) morpholino antisense oligonucleotides were purchased from GeneTools. In whole embryo experiments, synthetic mRNAs or antisense oligonucleotides were injected into the animal pole of one blastomere at the two-cell stage, or into the marginal zone of four-cell stage embryos (Fgf8aMO). Pax3GR- or Zic1GR-injected embryos were cultured in $0.1 \times$ normal amphibian medium

(NAM) containing $10 \mu \mathrm{M}$ dexamethasone from stage 10.5. Siblings injected with these constructs and cultured in the absence of dexamethasone were used as control. For animal explant experiments, both blastomeres of two-cell stage embryos were injected in the animal pole region, with Pax3GR, Zic1GR, or both, and explants were dissected at the late blastula stage and immediately cultured in vitro for several hours in NAM $0.5 \times$ plus $10 \mu \mathrm{M}$ dexamethasone (Dex Sigma-Aldrich, St. Louis, MO). In some experiments, $10 \mu \mathrm{g} / \mathrm{ml}$ cycloheximide (CHX; Sigma-Aldrich) was added to the culture medium to block protein synthesis, in which case explants were pretreated with cycloheximide for $30 \mathrm{~min}$ before dexamethasone treatment (Kolm and Sive, 1995). The animal explants were subsequently analyzed by real-time reverse transcription (RT)-PCR for the expression of various marker genes (Table 1).

\section{Lineage Tracing and Whole-Mount In Situ Hybridization}

In all experiments, embryos were coinjected with $\beta$-galactosidase ( $\beta$-gal) mRNA to identify the manipulated side. Embryos at the appropriate stage were fixed in MEMFA (Harland, 1991) and successively processed for RedGal (Research Organics, Cleveland, $\mathrm{OH}$ ) staining and in situ hybridization. Antisense digoxigenin-labeled probes (Genius kit; Roche Diagnostics, Indianapolis, IN) were synthesized using template cDNA encoding Xhe (Katagiri et al., 1997), Snail2 (Mayor et al., 1995), Six1 (Pandur and Moody, 2000), Sox2 (Mizuseki et al., 1998), Pax3 (Bang et al., 1997), and Zic1 (Mizuseki et al., 1998). Whole-mount in situ hybridization was performed as described previously (Harland, 1991). For histology, stained embryos were embedded into Paraplast,$+ 12-\mu \mathrm{m}$ sections were cut on a rotary microtome, and the sections were briefly counterstained with eosin.

\section{Real-Time RT-PCR}

For each sample, total RNAs were extracted from 10 animal explants by using an RNeasy microRNA isolation kit (QIAGEN, Valencia, CA) according to the manufacturer's direction. During the extraction procedure the samples were treated with DNase I, to eliminate possible contamination by genomic DNA. The amount of RNA isolated from tissues was quantified by measuring the OD using a spectrophotometer (Beckman Coulter, Fullerton, CA). Real-time RT-PCR was performed using the primers shown in Table 1 and the QuantiTect SYBR Green RT-PCR kit (QIAGEN) on LightCycler (Roche Diagnostics). The reaction mixture consisted of $10 \mu \mathrm{l}$ of QuantiTect SYBR Green RT-PCR Master Mix, $500 \mathrm{nM}$ forward and reverse primers, $0.2 \mu \mathrm{l}$ of RT, and $60 \mathrm{ng}$ of template RNA in a total volume of $20 \mu \mathrm{l}$. The cycling conditions were as follow: denaturation at $95^{\circ} \mathrm{C}(3$ s), annealing at $55^{\circ} \mathrm{C}(4 \mathrm{~s})$, and extension at $72^{\circ} \mathrm{C}(12 \mathrm{~s})$. By optimizing primers and reaction conditions, a single specific product was amplified as confirmed by melting curve analysis. Each reaction included a control without template and a 
Table 1. Primers used for real-time RT-PCR

\begin{tabular}{|c|c|c|}
\hline Gene & Forward primer & Reverse primer \\
\hline Pax3 & 5'-GCTGTGTCTGATCCAAGCAA-3' & 5'-CAATAGGCCGAACTGCTCTC-3' \\
\hline Zic1 & 5'-GTTGGAAGACTTGCCACCAT-3' & 5'-ACCTCTCGATCCGTCACATC-3' \\
\hline Msx1 & 5'-GCTCCСТCAACСТCACAGAG-3' & 5'-CATGGGTTTAGCAGCCATTT-3' \\
\hline Snail1 & 5'-TCACAAAGGCAGTGCTTCAC-3' & 5'-TTGTTCTCTGTGCCAACTGC-3' \\
\hline Snail2 & 5'-CATGGGAATAAGTGCAACCA-3' & 5'-AGGCACGTGAAGGGTAGAGA-3' \\
\hline Sox8 & 5'-AAGGTCTCTGGTGGCTGAAA-3' & 5'-CACCGCCACATTTCAGAGTA-3' \\
\hline Twist & 5'-GGGAGAAAATGATGCAGGAA-3' & 5'-TCACTGAGATCGGACTGTCG-3' \\
\hline c-Myc & 5'-CCGAAGAGAACGACAAGAGG-3' & 5'-AGAAATGGCGTATTCCGTTG-3' \\
\hline Xhe & 5'-CATGTCTAATGGCGGTTGTG-3' & 5'-TGCTGGATGATCCCCATATT-3' \\
\hline Crisp & 5'-AGTGAGGGCGAATTTTTCAA-3' & 5'-AACATTATTTCCCGCTGTGC-3' \\
\hline Six1 & 5'-CTGGAGAGCCACCAGTTCTC-3' & 5'-AGTGGTCTCССССТCAGTTT-3' \\
\hline Eya1 & 5'-ATGACACCAAATGGCACAGA-3' & 5'-GGGAAAACTGGTGTGCTTGT-3' \\
\hline Ncam & 5'-GAGCTGGTTTCTTCCACTGC-3' & 5'-GTTGGGGACTGTTTTGACT-3' \\
\hline Sox2 & 5'-ТСАССТСТTСТTСССАТTCG-3' & 5'-CGACATGTGCAGTCTGCTTT-3' \\
\hline Xcg & 5'-GAAGTAACATCAAACAAAGC-3' & 5'-GGTTGATGTTACTTCCCCAG-3' \\
\hline Keratin & 5'-CACCAGAACACAGAGTAC-3' & 5'-CAАССТТСССАТСАACСA-3' \\
\hline $\mathrm{EF} 1 \alpha$ & 5'-ACCCTCCTCTTGGTCGTTTT-3' & 5'-TTTGGTTTTCGCTGCTTTCT-3' \\
\hline
\end{tabular}

standard curve of serial dilution points (in steps of 10-fold) of test RNAs. In each case, elongation factor $1 \alpha(\mathrm{EF} 1 \alpha)$ was used as an internal reference (data not shown), and each bar on the histograms has been normalized to the level of EF1 $\alpha$ expression. The histograms in each figure are representative cases of at least three independent experiments.

\section{RESULTS}

\section{Molecular Identity of NPB Cells}

In Xenopus, three cell populations emerge at the NPB: the $\mathrm{NC}$, the PE, and the HG (Figure 1, A and B). A number of well-characterized molecular markers can be used to visualize these three cell types shortly after their induction (Figure $1 \mathrm{C}$ ). At the midneurula stage (stage 15), the NC and the PE express the transcription factors Snail2 and Six1, respectively, whereas cells of the prospective HG express the hatching enzyme (Xhe; Figure 1C). Double in situ hybridization (Figure 1D) and tissue sections (Figure 1E) reveal that Snail2, Six1, and Xhe have nonoverlapping expression domains at the neurula stage. Xhe is confined to the superficial layer of the ectoderm slightly medial to the Snail2 expression domain (Figure 1, D and E). Snail2 and Six1 are both restricted to the inner layer of the ectoderm; however, Six 1 abuts the neural plate at the most anterior region, and it is positioned lateral to the Snail2 expression domain (Figure 1, C and E). The expression pattern of these cell typespecific genes relative to the neural plate (visualized by Sox 2 expression; Figure 1C) is schematized in Figure 1F.

\section{Regulation of Cell Fates at the NPB by Bmp Attenuation and Wnt Signaling}

To analyze the regulation of cell fates at the NPB, embryos were injected with increasing doses of Noggin mRNA alone (to modulate the level of Bmp activity) or in combination with Wnt1 mRNA, because both factors have been shown regulate cell fate in the ectoderm (for review, see Knecht and Bronner-Fraser, 2002; Wu et al., 2003; Huang and SaintJeannet, 2004). The corresponding animal explants were isolated at the blastula stage, cultured for $5 \mathrm{~h}$ (equivalent stage $11.5)$ or $10 \mathrm{~h}$ (equivalent stage 14), and analyzed by real-time RT-PCR for the expression of genes expressed in various domains of the ectoderm. These genes include four transcription factors expressed early and broadly at the NPB, Pax3 (Bang et al., 1997), Zic1 (Mizuseki et al., 1998), Snail1 (Essex et al., 1993), and Msx1 (Suzuki et al., 1997); the NC- specific genes Snail2 (Mayor et al., 1995), Sox8 (O'Donnell et al., 2006), Twist (Hopwood et al., 1989), and cMyc (Bellmeyer et al., 2003); two genes expressed in the PE, Six1 (Pandur and Moody, 2000) and Eya1 (David et al., 2001); Xhe (Katagiri et al., 1997) and Crisp (Schambony et al., 2003) specific for HG; two neural plate marker genes, Sox2 (Mizuseki et al., 1998) and Ncam (Kintner and Melton, 1987); and finally, Keratin (Jonas et al., 1989) and Xcg (Jamrich and Sato, 1989), used to evaluate epidermis and cement gland formation, respectively. Results of a representative experiment are presented in Figure 2.

Among all the genes analyzed, Pax3, Zic1, and Snail1 were the only factors to show a significant activation after $5 \mathrm{~h}$ in culture (Figure 2A), consistent with their predicted function as NPB specifiers (Meulemans and Bronner-Fraser, 2004). Pax3 and Snail1 were both induced by the coinjection of Noggin and Wnt1, whereas Zic1 induction occurred as a result of Bmp attenuation, independent of the presence of Wnt1 (Figure 2A), suggesting that Pax3/Snail1 and Zic1 may regulate distinct fate at the NPB. Msx1, another predicted NPB specifier (Meulemans and Bronner-Fraser, 2004), was only weakly induced by coinjection of Noggin and Wnt1 after $5 \mathrm{~h}$ in culture. As previously reported, induction of both NC- (Snail2, Sox8, Twist, and c-Myc) and HG (Xhe and Crisp)-specific genes required Bmp attenuation as well as active Wnt signaling (Saint-Jeannet et al., 1997; LaBonne and Bronner-Fraser, 1998; McGrew et al., 1999). In contrast to Pax3, Snail1 and Zic1, these genes were only activated after $10 \mathrm{~h}$ in culture (Figure 2, B and C). The gene c-Myc was not only activated by the Noggin/Wnt combination, but also by injection of Noggin alone, which may reflect c-Myc expression in domains of the ectoderm other than the NC (Bellmeyer et al., 2003). Interestingly, we found that NC- and HG-specific genes were activated for different levels of Bmp activity in the presence of Wnt1. Strong induction of Snail2, Sox8, and Twist was observed with injection of $0.1 \mathrm{ng}$ of Noggin mRNA, whereas induction of Xhe and Crisp required at least $0.4 \mathrm{ng}$ of Noggin (Figure 2, B and C), indicating that the generation of $\mathrm{NC}$ and $\mathrm{HG}$ fates can be uncoupled experimentally. The PE-specific genes, Six1 and Eya1, were moderately but significantly induced by intermediate levels of Bmp activity (0.4 ng of Noggin) after $10 \mathrm{~h}$ in culture. As expected, the activation of early (Sox2) and late (Ncam) neural plate-specific genes was the strongest for the higher 
A

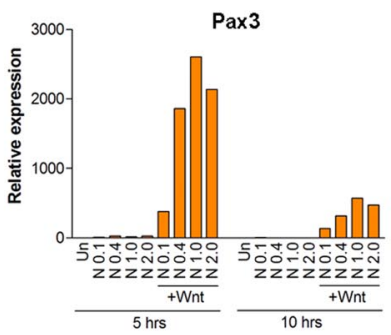

B

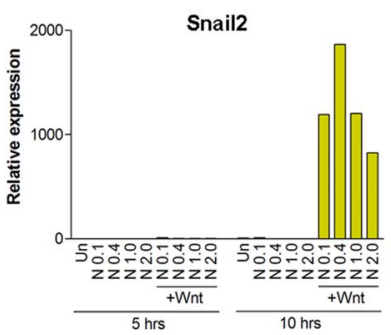

C

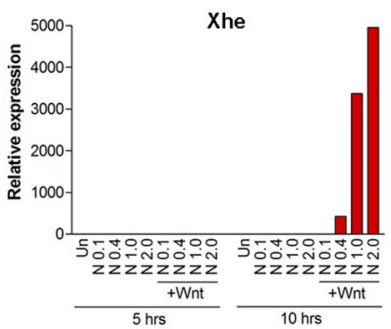

E

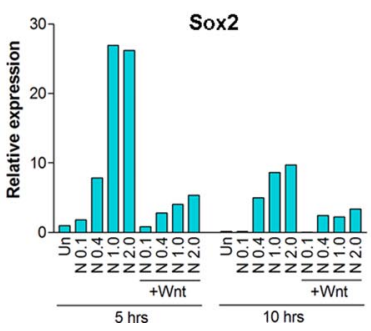

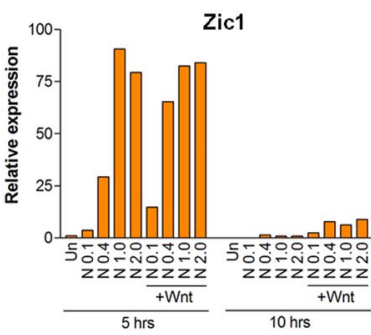
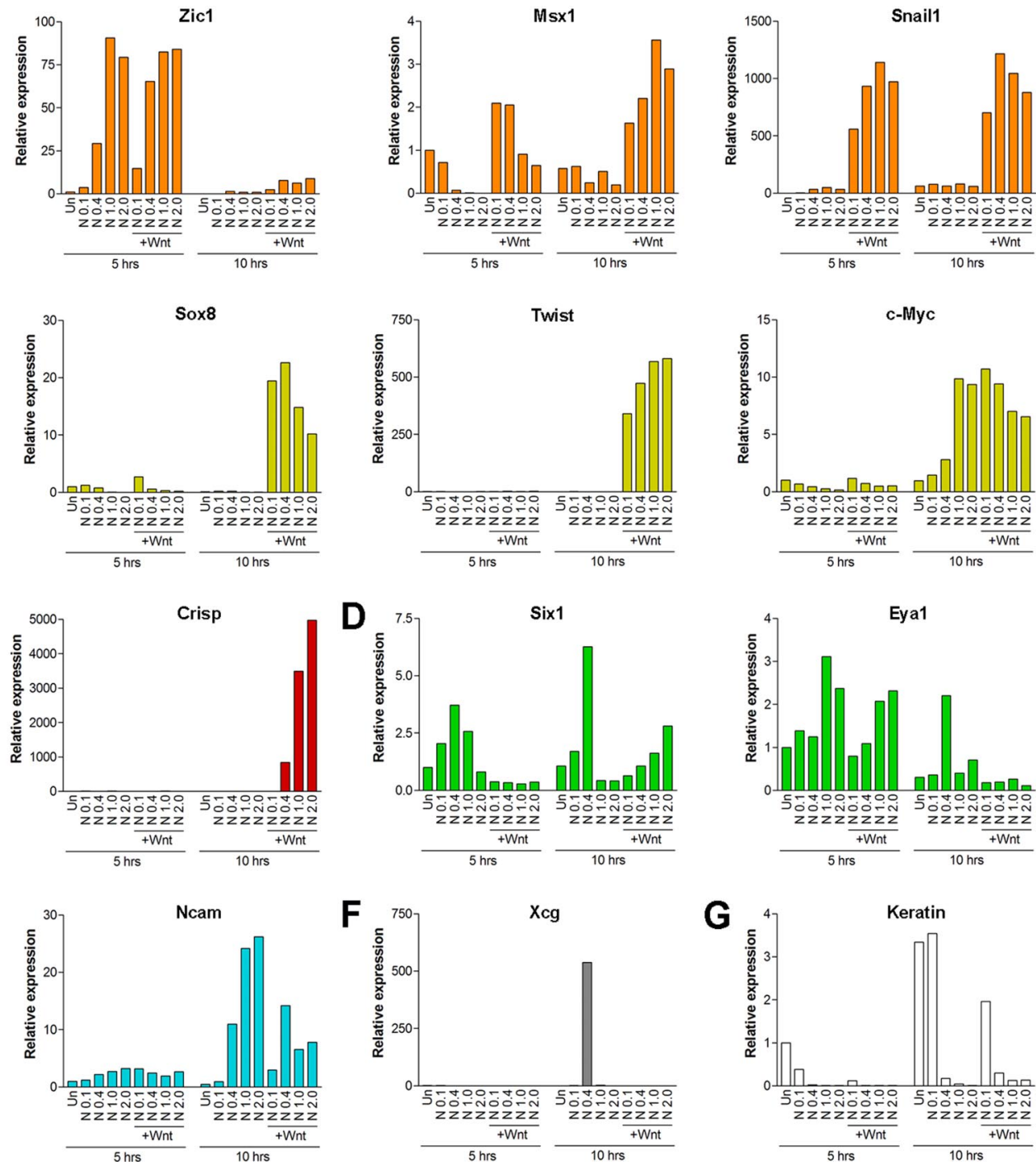

Figure 2. Regulation of NPB cell fates by Bmp attenuation and Wnt signaling. Embryos at the two-cell stage were injected in the animal pole with increasing doses of Noggin $(\mathrm{N})$ mRNA $(0.1,0.4,1.0$, and $2.0 \mathrm{ng})$ alone or in combination with Wnt1 (+Wnt) mRNA (100 pg). Animal explants were dissected at the blastula stage, cultured for 5 or $10 \mathrm{~h}$, and analyzed by real-time RT-PCR for the expression of four putative NPB specifiers, Pax3, Zic1, Msx1, and Snail1 (A), and a number of cell type-specific genes: Snail2, Sox8, Twist, and cMyc for NC (B); Xhe and Crisp for HG (C); Six1 and Eya1 for PE (D); Sox2 and Ncam for neural plate (E); Xcg for cement gland (F); and Keratin for epidermis (G). Un, uninjected animal explant. Each value was normalized to the level of EF1 $\alpha$ expression.

doses (1 ng and $2 \mathrm{ng}$ ) of Noggin mRNA (Figure 2E), and it was associated with strong repression of epidermal fate (Figure 2G). The cement gland, an embryonic structure developing anterior to the neural plate, was also induced in Noggin-injected explants as described previously (Lamb et al., 1993); however, compared with the neural plate marker Ncam, Xcg was activated for a single dose of Noggin mRNA (0.4 ng; Figure 2F).

These observations indicate that Bmp activity alone or in combination with Wnt signaling is implicated in the generation of multiple cell types in the ectoderm, including HG, $\mathrm{NC}, \mathrm{PE}$, neural plate, epidermis, and cement gland. Each one of these cell populations seems to require very specific thresholds of Bmp activity. Because of their early activation in this assay, Pax3, Snail1, and Zic1 are likely to be important early regulators of cell fate at the NPB. We decided to further analyze the role of two of these factors, Pax3 and Zic1, because Snail1 has been the focus of a previous study (Aybar et al., 2003).

\section{Developmental Expression of Pax3 and Zic1 at the NPB}

Pax3 and Zic1 are broadly expressed at the NPB. Their expression is initiated at the early to midgastrula stage (Figure $3 \mathrm{~A}$ ), before the onset of expression of Xhe, Snail2, and Six1. Pax3 and Zic1 are both expressed in a domain lateral to the neural plate. Zic1 expression domain extends more rostrally than Pax3 to delineate the anterior most region of the neural plate (Figure 3A). This region corresponds to the prospective PE, domain of expression of Six1 (Figure 1C). Zic1 expression also infringes onto the most lateral region of the neural plate (Figure 3A; Misuzeki et al., 1998). Tissue sections indicate that Pax3 and Zic1 are coexpressed in the inner layer of the ectoderm, prospective NC forming region, and Snail2 expression domain (Figure 1C). In the most superficial layer of the ectoderm, Pax3 is coexpressed with Xhe (Figure 3B), and later, at the tailbud stage, Xhe and Pax3 have similar expression domain in the developing HG (Figure 3D). The expression of Pax3 and Zic1 at the NPB is 

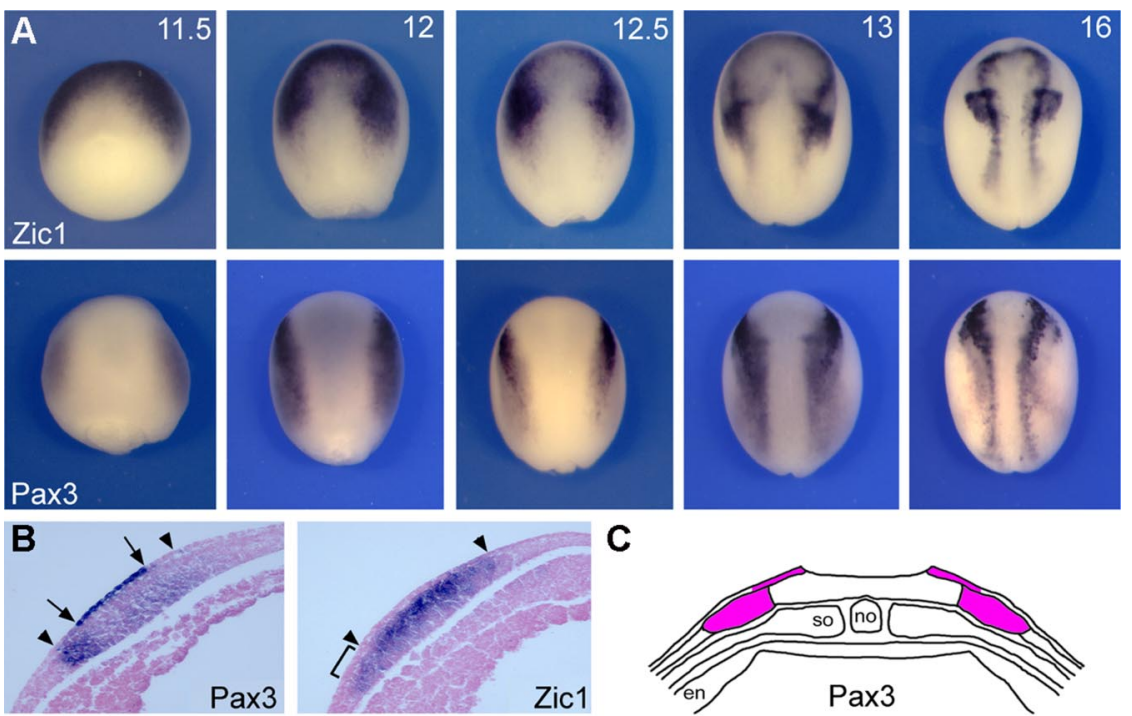

C
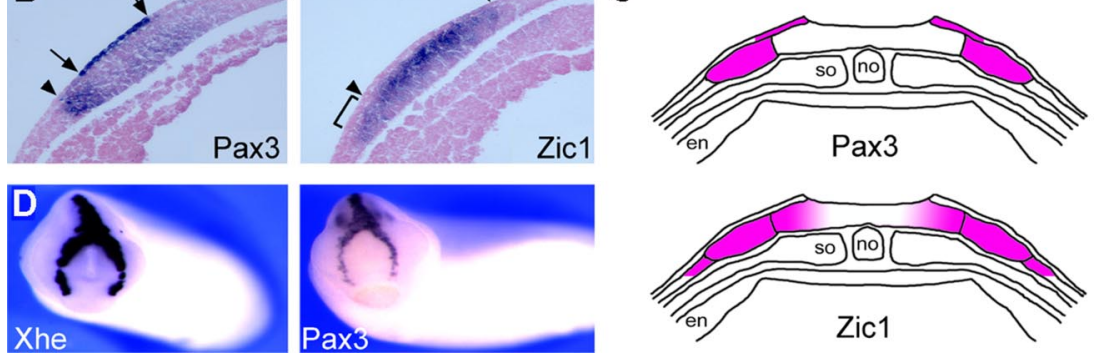

Figure 3. Expression of Pax3 and Zic1 at the NPB. (A) Developmental expression of Pax3 and Zic1 at the NPB in stage-matched embryos (the stages are indicated in the top right corner). Dorsal views, anterior to top. (B) Transverse section through neurula stage embryos illustrating the relative position of Pax3 and Zic1 in the deeper layer of the ectoderm (arrowheads). Pax3 is also detected in the superficial layer of the ectoderm (arrows), and Zic1 expression domain expands more laterally (bracket) than Pax3. (C) Schematic representation of cross-sections through neurula stage embryo showing the domain of expression of the Pax3 and Zic1 (purple). (D) Xhe and Pax 3 are coexpressed in the HG at the tailbud stage. Frontal views. schematized in Figure 3C. The early and broad expression of Pax3 and Zic1 at the NPB in regions of the ectoderm from which NC, HG, and PE arise is consistent with an early function of these factors in regulating the development of these three cell populations.

\section{Regulation of Cell Fates at the NPB by Fgf8a}

Because Fgf8 has been implicated in NC (Monsoro-Burq et al., 2003) and PE (Litsiou et al., 2005; Ahrens and Schlosser, 2005) formation, we decided to analyze the regulation of these NPB genes by Fgf8. Embryos were injected with Fgf8a mRNA in one blastomere at the two-cell stage and analyzed for the expression of Pax3, Zic1, Snail2, Xhe, and Six1 by whole mount in situ hybridization. Fgf8a correspond to a splice form of Fgf8 that has no mesoderm-inducing activity (Fletcher et al., 2006). Increasing doses of Fgf8a resulted in two major outcomes. Whereas low doses of Fgf8a mRNA $(0.1-0.5 \mathrm{pg})$ resulted in an expansion of most markers (Pax3, Zic1, Snail2, and Xhe) on the injected side (Figure 4A), high doses (5-50 pg) caused both a loss of expression of these markers on the injected side and an increase of the same markers on the controlateral side (Figure 4A). The later phenotype is presumably resulting from the diffusion of the Fgf8a protein. Excess Fgf8a signaling in the embryo is incompatible with the expression of the NPB genes. Although expression of Pax3, Zic1, Snail2, Xhe, and Six1 seemed to follow a similar pattern in response to exogenous Fgf8a, we also noticed some differences in the doses of Fgf8a for which these responses were observed. For example, inhibition of Six1 expression was observed unilaterally or bilaterally for doses of Fgf8a that resulted in expansion of Pax3, Zic1, Snail2, and Xhe on the injected side $(0.5 \mathrm{pg})$ or on the contralateral side ( $5 \mathrm{pg}$ ). These results indicate that genes at the NPB are extremely sensitive to variations in Fgf8a signaling and that each cell type may respond to specific thresholds of Fgf8a activity. The strong and uniform reduction of all five markers observed for the higher dose of Fgf8a (50 pg) is likely to be secondary to the posteriorizing activity of Fgf8 on the ectoderm, as reported previously (Fletcher et al., 2006).

To further evaluate Fgf8a requirement for the development of these fates, we performed a knockdown of Fgf8a by using a splice-blocking morpholino, the specificity of which has been well established (Fgf8aMO; Fletcher et al., 2006). Morpholinos injections were targeted to the marginal zone at the four-cell stage, one of the primary sources of Fgf8a at the gastrula stages (Christen and Slack, 1997). Pax3 was reduced in a large proportion of Fgf8aMO-treated embryos $(91 \%$; $n=56)$, whereas Zic1 anterior NC domain was expanded $(83 \% ; n=54)$. However, this anterior expansion was often associated with a loss of the most posterior domain of Zic1 (Figure 4B). The expressions of Snail2 (95\%; $n=56)$, Xhe $(85 \% ; n=27)$, and Six1 $(65 \%$; $n=43)$ were all significantly reduced in the absence of Fgf8a function (Figure 4B). Together, these results indicate that Fgf8a is a major regulator of cell fates at the NPB and that individual cell populations in this region of the ectoderm arise in response to sharp thresholds of Fgf8a activity.

\section{Pax3 Directly Regulates HG Fate}

Because Pax3 is expressed in the developing HG in addition to its NC expression domain (Figure 3, A, B, and D), we wanted to determine whether Pax3 had also a function in regulating $\mathrm{HG}$ formation in addition to its well-described role in NC development (for review, see Chi and Epstein, 2002). To this end, we generated a hormone-inducible construct in which Pax3 was fused to the hormone-binding domain of human glucocorticoid receptor (Pax3GR). The activity of such fusion proteins can be regulated by addition of dexamethasone in the culture medium of whole embryos or animal explants (Kolm and Sive, 1995). Embryos injected with $1 \mathrm{ng}$ of Pax3GR mRNA and treated with dexamethasone at the gastrula stage (stage 10.5-11) seemed to develop normally; however, premature hatching was observed in Pax3GR-injected embryos compared with uninjected siblings (Figure 5A), or injected embryos cultured in the ab- 


\section{A \\ Pax3}
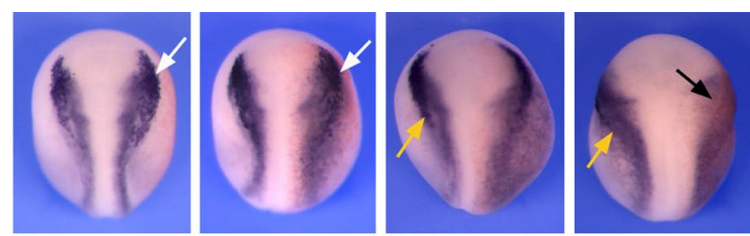

B
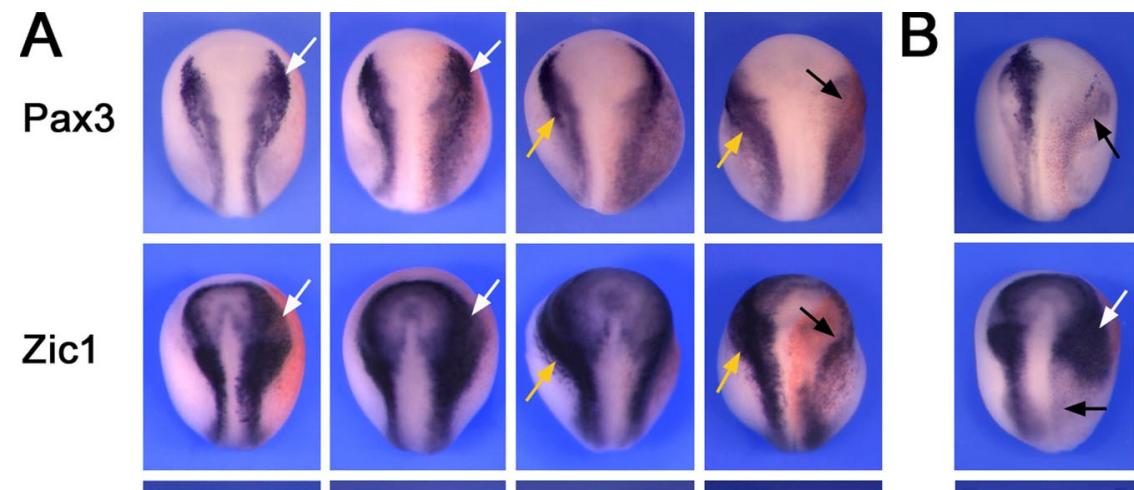

increasing amounts of Fgf8a mRNA $(0.1,0.5$ 5 , and $50 \mathrm{pg}$ ) in one blastomere at the two-cell stage and analyzed by whole-mount in situ hybridization for Pax3, Zic1, Snail2, Xhe, and Six1 expression. Pax3, Zic1, Snail2, and Xhe are expanded on the injected side by lower doses of Fgf8 ( 0.1 or $0.5 \mathrm{pg}$; white arrows). The expression of these genes is expanded on the contralateral side (yellow arrows) and repressed on the injected side (black arrows) for higher doses of Fgf8a (5 or $50 \mathrm{pg}$ ). Six1 expression is repressed unilaterally or bilaterally for intermediate $(0.5$ pg) or high (5 and $50 \mathrm{pg}$ ) doses of Fgf8a, respectively. (B) Embryos injected with Fgf8a-specific morpholino (Fgf8aMO) in the marginal zone of four-cell stage embryos exhibit a strong reduction of Pax3, Snail2, Xhe, and Six1 expression at the neurula stages (black arrows). In the absence of Fgf8a function, Zic1 anterior expression domain is expanded (white arrow), whereas posteriorly Zic1 is reduced (black arrow). In A and B, embryos hybridized with Pax3, Zic1, and Snail2 are viewed from the dorsal side, anterior to top. Anterior views are shown for embryos hybridized with Xhe and Six1. In all panels, the injected side is on the right.
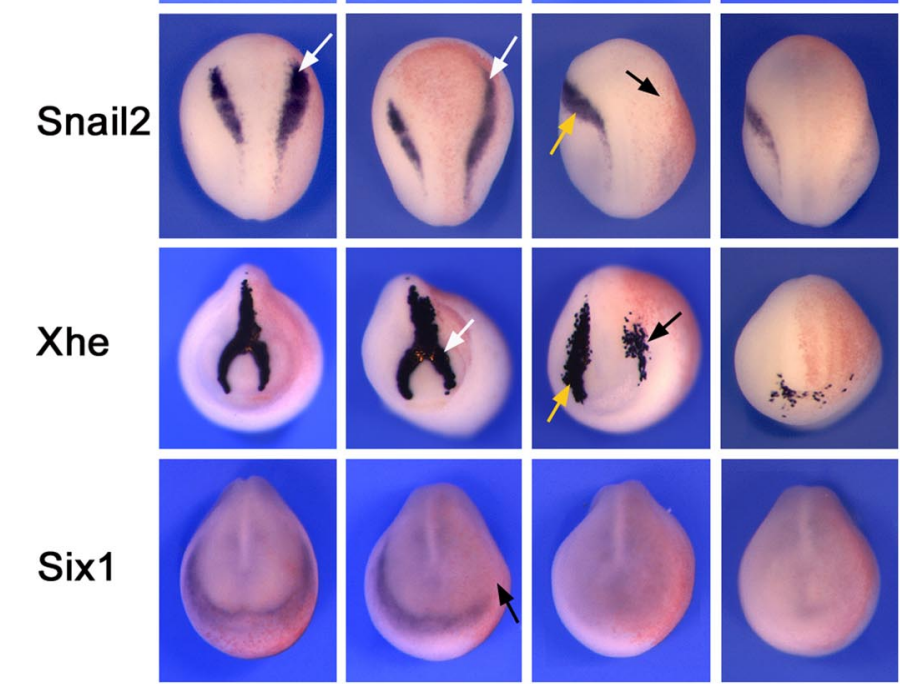

$0.1 \mathrm{pg}$

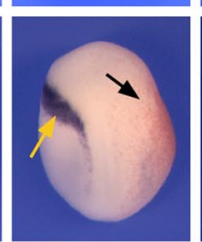

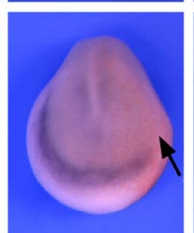

$0.5 \mathrm{pg}$

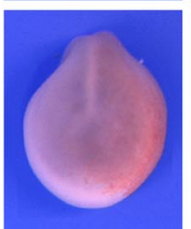

$5 \mathrm{pg}$

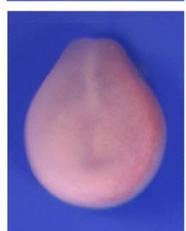

$50 \mathrm{pg}$
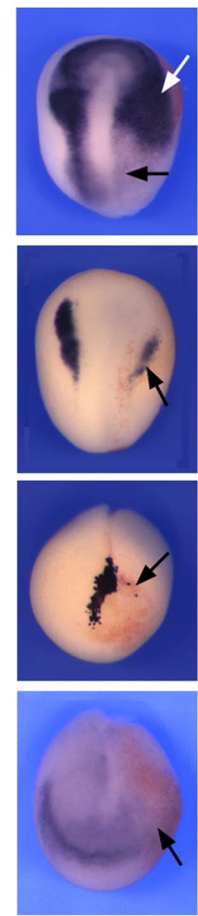

Fgf8aMO sence of dexamethasone (data not shown). The vitelline membrane of these embryos breaks open at stage 25 , several hours earlier than in wild-type embryos, in which hatching normally occurs around stage 30-32 (Nieuwkoop and Faber, 1967). Consistent with this observation we found that Pax3GR-injected embryos showed strong ectopic expression of Xhe on the injected side (Figure 5B), suggesting that Pax3 controls premature hatching by up-regulation of Xhe, the enzyme involved in degradation of the vitelline envelope. Sibling embryos injected with Pax3GR and cultured in the absence of dexamethasone had normal Xhe expression (Figure 5B).

We also analyzed the regulation of HG fate by Pax3 in animal caps. Explants isolated from blastula-stage embryos injected with Pax3GR at the two-cell stage were cultured in vitro for $4 \mathrm{~h}$ in the presence of dexamethasone and analyzed by real-time RT-PCR. Pax3GR induced strong expression of Xhe and Crisp. Interestingly, although Crisp activation was dramatically reduced by cycloheximide treatment (+CHX), Pax3-mediated activation of Xhe occurred independently of protein synthesis (Figure 5C). These results indicate that Pax3 regulates $\mathrm{HG}$ by directly activating Xhe and indirectly regulating Crisp.

\section{Pax3 Activity Is Required for Both NC and HG Formation}

To analyze the requirement for endogenous Pax3 activity in HG induction, we used a Pax3-specific morpholino oligonucleotide (Pax3MO), which blocks Pax3 translation as de- scribed previously (Monsoro-Burq et al., 2005). When injected in one blastomere at the two-cell stage, Pax3MO inhibited Snail2 expression at the neurula stage ( $89 \%$ of the embryos; $\mathrm{n}=52$ ), as reported previously (Monsoro-Burq et al., 2005; Sato et al., 2005), but it also prevented Xhe expression $(92 \%$ of the embryos; $n=59)$ at the tailbud stage (Figure 5D). Concomitant to the loss of Snail2 and Xhe expression, the neural plate gene Sox 2 was slightly expanded in $48 \%$ of the embryos $(n=42)$. In these embryos lacking Pax3 function, Zic1 expression domain was expanded (79\% of the embryos; $n=42$; Figure 5D), suggestive of a cross-regulation between these two NPB specifiers.

Because coinjection of Noggin and Wnt1 leads to an early activation of Pax3 (Figure 2A) and subsequent induction of NC and HG marker (Figure 2, B and C), we analyzed the dependence of Snail 2 and Xhe induction on Pax3 function. We found that Pax3MO strongly inhibited Snail2 and Xhe induction in explants treated by Noggin and Wnt1 (Figure $5 \mathrm{E})$. Together, these results demonstrate that Pax3 is required for both NC and HG formation downstream of NPBinducing signals, establishing a direct link between Pax3 and the specification of NC and HG fates.

\section{Distinct Thresholds of Pax3 Activity Regulate HG and NC Fates}

We found that Pax3 was able to increase NC progenitors formation based on the expanded expression of Snail 2 and Sox10 (data not shown), consistent with previous reports 

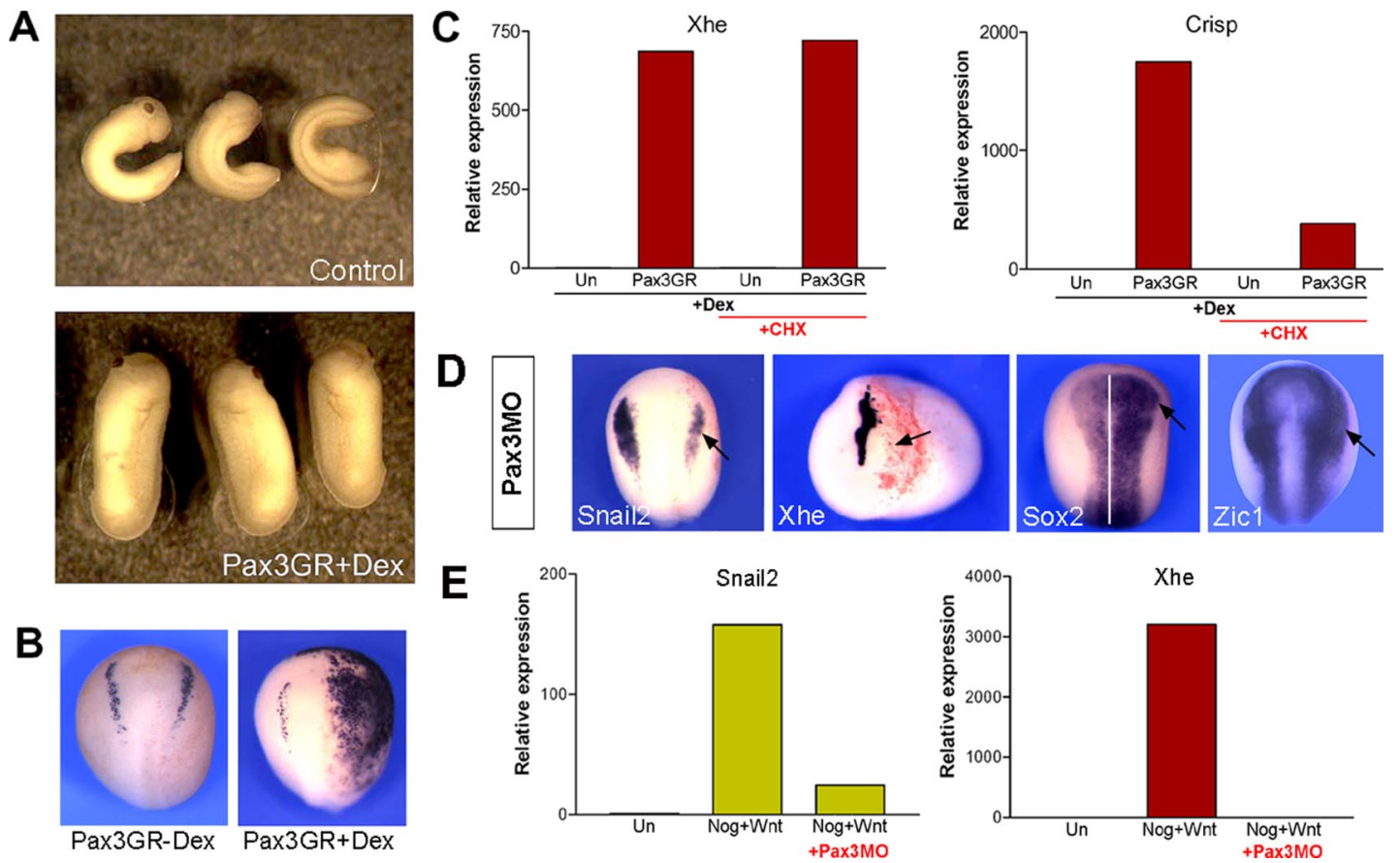

Figure 5. Pax3 is sufficient and required to promote HG fate. (A) Embryos injected with Pax3GR and treated with dexamethasone at the gastrula stage hatch several hours earlier than their uninjected siblings. (B) Stage 17 embryo that received unilateral injection of Pax3GR at the two-cell stage and treated with dexamethasone (+Dex) exhibit a strong ectopic expression of Xhe on the injected side (right side), whereas sibling embryos cultured in the absence of dexamethasone (-Dex) are unaffected. Dorsal views, anterior to top. (C) In animal explants, Pax3GR strongly induces Xhe and Crisp expression $4 \mathrm{~h}$ after dexamethasone treatment (+Dex). Pax3-mediated induction of Xhe occurs independently of protein synthesis (+CHX). Another HG-specific gene, Crisp, is significantly reduced in the absence of protein synthesis. (D) Embryos injected with Pax3 morpholino (Pax3MO) exhibit a strong reduction of Snail2 and Xhe expression at the neurula and tailbud stages, respectively (arrows). Sox2 and Zic1 expression were expanded in Pax3-depleted embryos (arrows). Embryos hybridized with Snail2, Sox2, and Zic1 are viewed from the dorsal side, anterior to top. For Xhe staining, anterior view is shown. In all cases, the injected side is indicated by an arrow. (E) Noggin +Wnt activates expression of Snail2 and Xhe in animal explants, an activity that is inhibited by coinjection of Pax3MO.

(Monsoro-Burq et al., 2005; Sato et al., 2005). However, we also noted that the same NC markers were sometimes strongly inhibited in Pax3-injected embryos, suggesting that in these embryos Pax3 may have induced the formation of another cell type at the expense of NC progenitors.

To evaluate this possibility, we injected increasing amounts of Pax3GR mRNA in two-cell stage embryos, and we analyzed the expression of NC (Snail2) and HG (Xhe) markers at stage 17. We found that although ectopic Xhe induction was strictly proportional to the amount of Pax3GR mRNA injected (Figure 6A), Snail2 was expanded by lower doses of Pax3GR (0.025-0.1 $\mathrm{ng}$ ) and strongly inhibited by higher doses (0.25-0.5 ng). In the absence of dexamethasone, embryos injected with Pax3GR showed normal Slug and Xhe expression (data not shown). These results suggest that NC and HG fate can coexist in the embryo up to a certain threshold of Pax3 activity and that high level of Pax3 activity converts the entire ectoderm into HG precursor cells (Figure 6A).

We confirmed these observations in animal explants by monitoring Snail2 and Xhe induction by real-time RT-PCR in response to increasing doses of Pax3GR mRNA. These explants were cultured in the presence of dexamethasone for $8 \mathrm{~h}$. As observed in vivo, high doses of Pax3GR strongly induces Xhe, whereas Snail2 expression requires intermediate doses of Pax3GR (Figure 6B). The same pattern of induction was observed for other NC and HG marker genes (Sox8 and Crisp; data not shown). That Snail2 and Xhe require different levels of Pax3 activity for their activation further supports the view that these cell types are induced independently (Figure 2, B and C).

It has been recently reported that Pax3 and Zic1 synergize to generate NC in Xenopus (Monsoro-Burq et al., 2005; Sato et al., 2005) consistent with the coexpression of these factors in the NC forming region (Figure 3, A and B). In animal explants coinjection of Pax3GR and Zic1GR elicited strong induction of Snail2, for doses of Pax3GR that normally induce only low level of Snail2 (Figure 6B), further establishing the importance of the cooperation between Pax 3 and Zic1 for the generation of NC progenitors. In these samples, the coinjection of Zic1GR also shifted the doses of Pax3GR required for $X$ he induction toward the higher end of the spectrum (Figure 6B). Together, these results indicate that distinct levels of Pax3 activity are involved in promoting either HG or NC fates and that Zic1 synergizes with Pax3 to elicit NC fate.

\section{Zic1 Is Sufficient to Activate PE Genes, an Activity That Is Repressed by Pax3}

Because Zic1 is expressed in a broad domain anterior to the neural plate that includes the PE (Figure $3 \mathrm{~A}$ ), and it is strongly activated by Bmp inhibition in the ectoderm (Figure $2 \mathrm{~A})$, we wanted to determine whether Zic1 regulates PE specification. To test this possibility, we analyzed the fate of animal explants injected with increasing amounts of Zic1GR mRNA and treated with dexamethasone immediately after dissection at the blastula stage. In these explants, a strong 
Figure 6. Distinct thresholds of Pax3 activity regulate HG and NC fates. (A) Embryos injected with increasing amounts of Pax3GR mRNA in one blastomere at the two-cell stage and treated with dexamethasone at the gastrula stage were analyzed by whole-mount in situ hybridization for Xhe and Snail2 expression at stage 17. Although Snail2 is expanded laterally and anteriorly by lower doses of Pax3GR, Snail2 is repressed for higher doses of Pax3GR. Ectopic expression of Xhe is proportional to the amount of Pax3GR mRNA injected. Dorsal views, anterior to top. In all panels, the injected side is on the right. (B) In animal explants, the induction of Snail2 and Xhe as determined by real-time RT-PCR depends on different amounts of injected Pax3GR. Zic1GR strongly synergizes with Pax3 to induce Snail2. The concentrations of Pax3GR mRNA injected are in nanograms. A single dose of Zic1GR mRNA $(0.25 \mathrm{ng})$ was coinjected as indicated.
A

Xhe
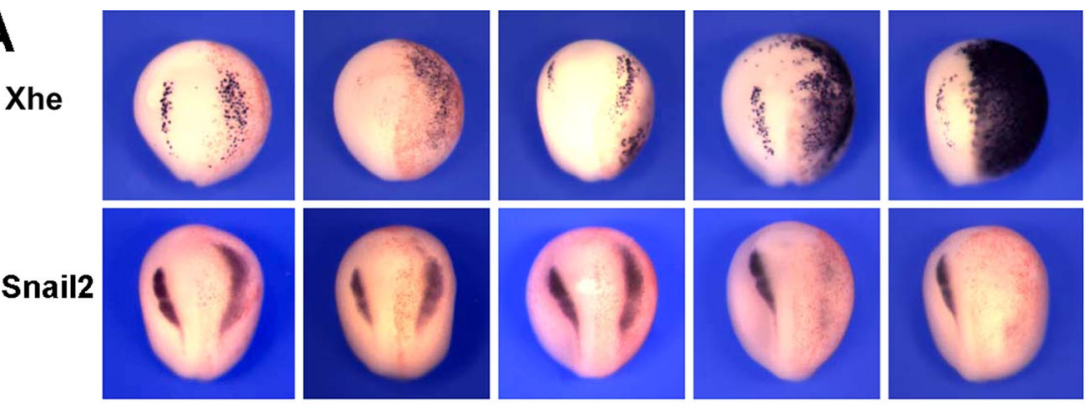

$0.025 n g$
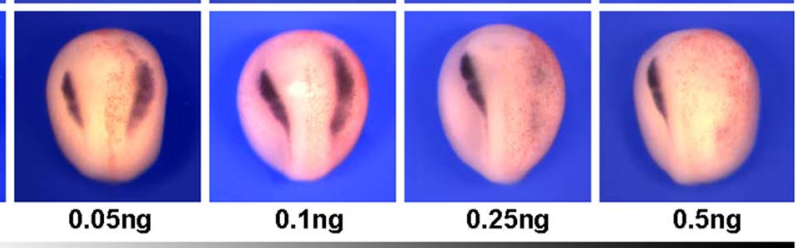

$0.1 \mathrm{ng}$

$0.25 n g$

$0.5 n g$

Pax3GR
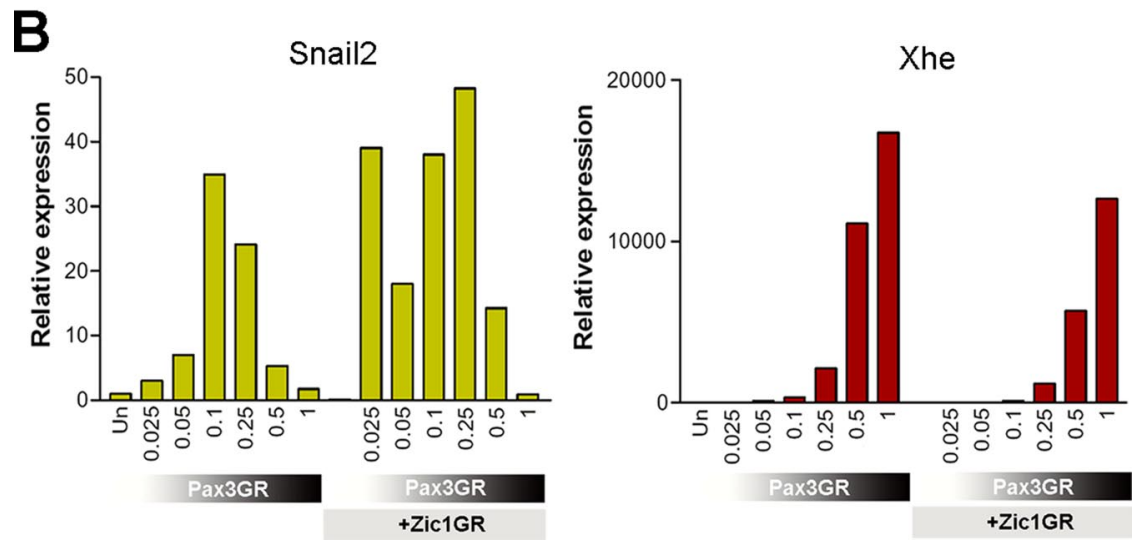

activation of the PE markers Six1 (Figure 7A) and Eya1 (data not shown) was observed in response to Zic1GR injection, and this induction was stronger for intermediate doses of Zic1GR mRNA (0.05-0.1 ng). This result points to an important role of Zic1 in promoting PE fate. Importantly, in these explants the coinjection of Pax3GR strongly repressed Zic1mediated induction of Six1 (Figure 6A) and Eya1 (data not shown) and promoted Snail2 and Xhe expression (Figure 7A). These observations indicate that NC and PE fates are exclusive from one another and further support the view that Zic1 and Pax3 cooperate to promote NC formation.

\section{Zic1 Is Required for NC and Lateral PE Specification}

To analyze the requirement for endogenous Zic1 activity in $\mathrm{PE}$ and NC fate specification, we injected 40-45 ng of a Zic1-specific morpholino oligonucleotide (Zic1MO) in one blastomere at the two-cell stage. The specificity of this morpholino has been well described (Sato et al., 2005). In the absence of Zic1 function, Snail2 expression was severally reduced in $95 \%$ of the embryos $(n=43)$ as reported previously (Monsoro-Burq et al., 2005; Sato et al., 2005; Figure 7B). We also found that Six1 expression $(75 \% ; n=74)$ and the preplacodal expression domain of Sox $2(89 \% ; n=59)$ were strongly inhibited in morpholino-injected embryos (Figure 7B). In most of these embryos, the neural pate expression domain of Sox2 was expanded on the injected side (Figure 7B). Interestingly, only the lateral preplacodal domain of Six1 was affected in these embryos, suggesting that the most anterior region of the PE might be regulated by a different set of factors. In embryos lacking Zic1 function, Pax3 expression domain seemed more diffuse and overall reduced in $93 \%$ of the embryos $(n=61$; Figure $7 \mathrm{~B})$. This observation further supports the view that Zic1 and Pax3 can regulate each other's expression (Figure 5D). This reduction in Pax3 expression in morpholino-treated embryos may explain why $48 \%$ of these embryos $(n=40)$ also showed a decrease in Xhe expression (Figure $7 \mathrm{~B}$ ).

Because treatment of animal explants by Noggin + / -Wnt results in an early activation of Zic1 and the subsequent induction of NC (Snail2 and Sox8) and PE (Six1 and Eya1) fates (Figure 2, B and D), we decided to determine whether these fates were dependent on Zic1 function in animal explants. In these preparations, noggin-mediated induction of Six1 was strongly inhibited by coinjection of Zic1MO (Figure 7C). Similarly, Snail2 induction in Noggin +Wnt-injected explants was abolished in the absence of Zic1 function (Figure 7C). Xhe expression was also reduced in Zic1-depleted explants that received injection of Noggin +Wnt (Figure 7C), presumably as an indirect consequence of the effect of the loss of Zic1 function on Pax3 expression (cf. Figure 7B). Together, these results indicate that Zic1 is required downstream of Bmp and Wnt signaling to initiate the development of both PE and NC fates.

\section{DISCUSSION}

In an assay designed to determine the timing and sequence of induction of NPB-specific genes in response to Bmp attenuation and Wnt signaling, we found that only a small number of genes showed an early activation after $5 \mathrm{~h}$ in culture, namely, Pax3, Zic1, and Snail1 (Figure 2A). Snail1 and its related family member Snail2 are often seen as largely interchangeable during NC development. However, both genes behaved quite differently in this assay; Snail1 is activated much earlier than Snail2, thereby positioning Snail1 as a NPB specifier. Consistent with this observation, Snail1 is one of the few transcription factors capable of inducing NC marker genes when expressed in isolated an- 

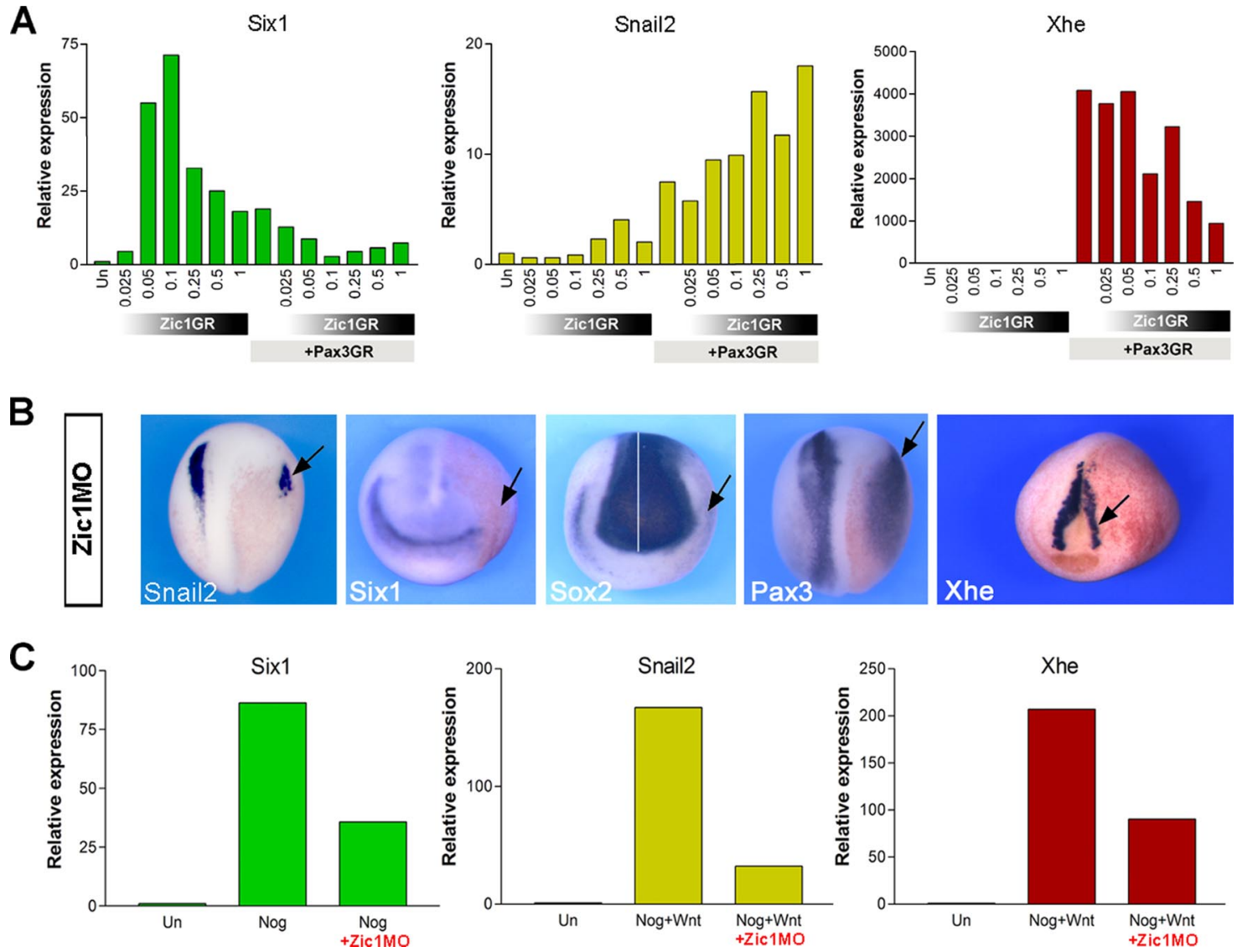

Figure 7. Zic1 promotes the expression of Six1 an activity repressed by Pax3. (A) In animal explants, Zic1GR is sufficient to activate Six1 expression as determined by real-time RT-PCR. Pax3GR strongly represses Zic1-mediated Six1 induction to promote NC (Snail2) and HG (Xhe) fates. The concentrations of Zic1GR mRNA injected are in nanograms. A single dose of Pax3GR mRNA (0.25 ng) was coinjected as indicated. (B) Embryos injected with Zic1 morpholino (Zic1MO) exhibit a strong reduction of Snail2 and Six1 (arrows). The neural plate expression of Sox2 is expanded, whereas Sox2 PE expression domain is reduced (arrow). In these embryos lacking Zic1 function, Xhe expression is altered (arrow), presumably as a consequence of reduced Pax3 expression (arrow). Embryos are viewed from the dorsal side, anterior to top (Snail2 and Pax3). In Six1, Sox2, and Xhe staining, anterior views are shown, dorsal to top. The injected side is indicated by an arrow. (C) Noggin-mediated induction of Six1 is inhibited in the absence of Zic1 function (+Zic1MO). Noggin + Wnt activates expression of Snail2 and Xhe in animal explants, an activity that is inhibited by coinjection of Zic1MO.

imal explants (Aybar et al., 2003). Although Pax3 and Snail1 are activated by the combination Noggin +Wnt, Zic1 is activated by Bmp attenuation, and this induction is unaffected by the presence of a Wnt signal. Because of this pattern of induction, Zic1 is expected to have unique characteristics but also to share some of the properties displayed by Pax3 or Snail1, at least in tissues in which these factors are coexpressed.

The early induction of these NPB specifiers was followed by the induction, after $10 \mathrm{~h}$ of culture, of a cohort of genes specific for the three lineages arising from the NPB: Xhe and Crisp for HG; Snail2, Sox8, Twist, and $\mathrm{cMyc}$, for NC; and Six1 and Eya1 for PE. The activation of NC- and HG-specific genes occurred in response to the same combination of signals (Noggin +Wnt), but for different levels of Bmp activity. In contrast, PE-specific genes were only activated in response to Bmp attenuation. An important point of this study is that we were able to establish a direct link between the induction of these cell types (NC, HG, and PE) specifiergenes and the early activation of Pax 3 and Zic1 mediated by $\mathrm{Bmp}+/-\mathrm{Wnt}$ signaling (Figures 5E and 7C). These results strongly support the proposed model of sequential activation of NPB genes (Meulemans and Bronner-Fraser, 2004; Litsiou et al., 2005).
Fgf is another signaling pathway with important patterning function in the developing embryo (for review, see Bottcher and Niehrs, 2005). Fgf8 is expressed in the paraxial mesoderm and in the anterior neural ridge (Christen and Slack, 1997), and it has been implicated in NC and PE formation (Villanueva et al., 2002; Monsoro-Burq et al., 2003; Ahrens and Schlosser, 2005; Litsiou et al., 2005). Varying the levels of Fgf8a signaling in the embryo revealed that NPB genes are highly sensitive to this factor (Figure 4A). Morpholino-mediated knockdown of Fgf8a resulted in a broad loss of NC-, HG-, and PE-specific genes, further supporting a requirement for Fgf8 function in the generation of NPB cell populations (Figure 4B). In contrast to a recent report (Monsoro-Burq et al., 2005), our results show that Pax3 expression at the NPB is not only dependent on a Wnt signal but also is tightly linked to Fgf8 signaling, suggesting that both signaling pathways converge at the level of Pax3 to generate NC. A similar positive influence of Fgf8 on Pax3 expression at the NPB has also been described in another study (Sato et al., 2005).

One of the most striking phenotypes of Pax3 gain function is a premature hatching of the embryo from its vitelline membrane (Figure 5A). We found that this phenotype is a direct consequence of the ability of $\mathrm{Pax} 3$ to activate tran- 
scription of Xenopus hatching enzyme, Xhe. This observation, together with the loss of Xhe expression in embryos lacking Pax3 function, establishes an essential role for Pax3 in $\mathrm{HG}$ development (Figure 5D). Although the function of Pax3 in NC and skeletal muscle formation is extremely well conserved during evolution (Chi and Epstein, 2002), the function of Pax3 in the development of the HG seems to be very unique to the Xenopus embryo. In the zebrafish, the HG has a different embryonic origin, it is derived from the endomesoderm, and neither Pax3 nor Pax7 is expressed in this tissue (Seo et al., 1998).

Using a knockdown approach in whole embryos and animal explants, we provide strong evidence that Pax3 is necessary for the regulation of both HG and NC fates (Figure 5, $\mathrm{D}$ and $\mathrm{E})$. Moreover, the gain of function experiments gave us some important clues regarding the molecular mechanisms underlying the regulation of these two fates by Pax3. In whole embryos, we observed that Snail2 induction depends on a specific level of Pax3 activity in the ectoderm, whereas Pax3-mediated activation of Xhe occurs in a strict dose-dependent manner. Moreover, excess Pax3 activity in the ectoderm is incompatible with NC development, but rather it promotes HG fate, and in the most extreme cases, it converted the entire ectoderm into Xhe-producing cells (Figure $6 \mathrm{~A}$; data not shown). These results indicate that varying the levels of activity of a single transcription factor (Pax3) in the ectoderm is sufficient to regulate the development two distinct cell fates at the NPB.

Several recent studies have established the cooperative activity of Pax3 and Zic1 in the control of NC formation in Xenopus (Monsoro-Burq et al., 2005; Sato et al., 2005). In regions of the ectoderm where Pax3 and Zic1 are coexpressed these factors synergize to promote $\mathrm{NC}$ fate. We also observed a cooperation of Zic1 and Pax3 in the induction of NC (Figures 6B and 7A). However, in contrast to these studies (Monsoro-Burq et al., 2005; Sato et al., 2005), we found that Pax3 expression can activate NC markers (Snail2 and Sox8) in animal explants (Figure 6B). This apparent discrepancy can be explained by differences in the amount of Pax3 injected in these explants, because we show that effect can only be obtained for intermediate level of Pax3 activity (Figure 6B). Our results do not imply that Pax3 alone is sufficient to generate NC; rather, we believe that in animal explants Pax3 cooperate with endogenous Zic1 (Mizuseki et al., 1998) to elicit NC fate. By varying the ratio of Pax3 and Zic1 in the ectoderm, we demonstrate that the synergistic activity of Pax3 and Zic1 can only be achieved when these two factors reach an appropriate balance (Figure 6B).

The dual activity of Pax3 in regulating both NC and HG fates raised the intriguing possibility that in regions of the ectoderm where it is not coexpressed with Pax3, Zic1 may also have the ability to regulate another cell fate. Zic1 is expressed in the anterior region of the embryo, lateral to the neural crest and at the most rostral boundary of the neural plate, prospective region of the PE. Although Zic1 is expressed in the ectoderm at the gastrula stage, these endogenous levels are clearly not sufficient to activate preplacodal fate when the ectoderm is isolated and cultured in vitro, suggesting that another factor might be required to maintain Zic1 expression in the ectoderm or to increase its expression to levels that are compatible with PE fate. Consistent with this view, we observed that Zic1-mediated induction of Six1 and Eya1 depends on a specific level of Zic1 activity in the ectoderm (Figure 7A). Interestingly, Pax3 has a strong inhibitory activity on Zic1-mediated induction of PE specifiers (Six1 and Eya1). This loss of PE fate is associated with a potent induction of NC- (Snail2) and HG (Xhe)-specific
A

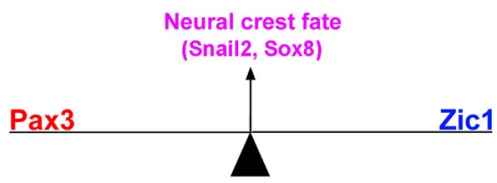

B Zic1
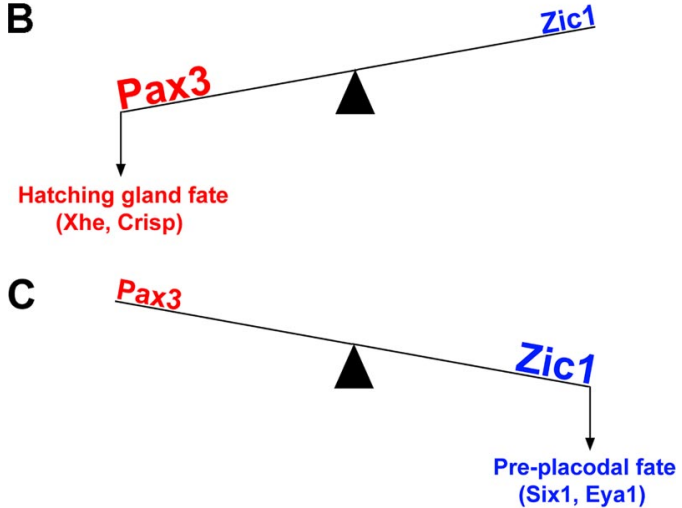

Figure 8. Model for the specification of NPB cells. In response to Bmp, Wnt, and Fgf signaling, the transcription factors Pax3 and Zic1 are activated at the NPB. The activity of these two factors regulates the specification of three NPB cell populations. When Pax3 and Zic1 reach an appropriate balance, $\mathrm{NC}$ is induced (A). Variations in the relative activity of these two transcriptional regulators at the NPB can disrupt this balance, either toward Pax3 to promote HG formation (B) or toward Zic1 to promote PE fate (C).

genes (Figure 7A). The inhibitory activity of Pax3 could be seen as a molecular mechanism by which the PE is restricted lateral to the $\mathrm{NC}$ forming region in the developing embryo. Embryos lacking Zic1 function have a reduction of Snail2 expression as reported previously (Monsoro-Burq et al., 2005; Sato et al., 2005), but they also show a loss of the lateral PE expression domain of Six1 and Sox2 (Figure 7B), further supporting an essential role of Zic1 in the establishment of PE fate. In these embryos, the most anterior PE domain was largely unaffected, suggesting that the most anterior subdomain of the PE is regulated independently of Zic1 function.

In this study, we provide novel information on the molecular mechanisms regulating cell fates at the NPB. In response to signaling events mediated by molecules of the Bmp, Wnt, and Fgf families, Pax3 and Zic1 are activated in distinct and overlapping domains of the ectoderm. The differential activity of these two NPB specifiers participates in the specification of all three NPB cell types in Xenopus (Figure 8). Although in distinct regions of the ectoderm, Pax3 and Zic1 can independently promote HG and PE fates; in domain of the ectoderm where both factors are coexpressed, their combined activities specify the NC. Furthermore, the tight regulation of Pax3 and Zic1 expression at the NPB establishes a balance between these two factors critical to NC development (Figure 8A). Variations in the relative activity of these two transcriptional regulators can disrupt this balance, either toward Pax3 to promote HG formation (Figure $8 \mathrm{~B}$ ) or toward Zic1 to promote PE fate (Figure $8 \mathrm{C}$ ).

\section{ACKNOWLEDGMENTS}

We are grateful to Dan Kessler for comments on the manuscript, to Richard Behringer for discussions, to Christine Credidio and Beth Aksim for technica assistance, and to Thomas Sargent for reagents. This work was supported by National Institutes of Health grant DE-14212. 


\section{REFERENCES}

Aybar, M. J., Nieto, M. A., and Mayor, R. (2003). Snail precedes slug in the genetic cascade required for the specification and migration of the Xenopus neural crest. Development 130, 483-494.

Ahrens, K., and Schlosser, G. (2005). Tissues and signals involved in the induction of placodal Six1 expression in Xenopus laevis. Dev. Biol. 288, 40-59.

Bang, A. G., Papalopulu, N., Kintner, C., and Goulding, M. D. (1997). Expression of Pax-3 is initiated in the early neural plate by posteriorizing signals produced by the organizer and by posterior non-axial mesoderm. Development 124, 2075-2085.

Bellmeyer, A., Krase, J., Lindgren, J., and LaBonne, C. (2003). The protooncogene c-myc is an essential regulator of neural crest formation in Xenopus. Dev. Cell 4, 827-839.

Bottcher, R. T., and Niehrs, C. (2005). Fibroblast growth factor signaling during early vertebrate development. Endocrine Rev. 26, 63-77.

Brugmann, S. A., and Moody, S. A. (2005). Induction and specification of the vertebrate ectodermal placodes: precursors of the cranial sensory organs. Biol. Cell 97, 303-319.

Brugmann, S. A., Pandur, P. D., Kenyon, K. L., Pignoni, F., and Moody, S. A. (2004). Six1 promotes a placodal fate within the lateral neurogenic ectoderm by functioning as both a transcriptional activator and repressor. Development $131,5871-5881$

Chi, N., and Epstein, J. A. (2002). Getting your Pax straight: Pax proteins in development and disease. Trends Genet. 18, 41-47.

Christen, B., and Slack, J.M.W. (1997). FGF-8 is associated with anteroposterior patterning and limb regeneration in Xenopus. Dev. Biol. 192, 455-466.

David, R., Ahrens, K., Wedlich, D., and Schlosser, G. (2001). Xenopus Eya1 demarcates all neurogenic placodes as well as migrating hypaxial muscle precursors. Mech. Dev. 103, 189-192.

Drysdale, T. A., and Elinson, R. P. (1991). Development of the Xenopus laevis hatching gland and its relationship to surface ectoderm patterning. Development 111, 469-478.

Essex, L. J., Mayor, R., and Sargent, M. G. (1993). Expression of Xenopus Snail in mesoderm and prospective neural fold ectoderm. Dev. Dyn. 198, 108-122.

Fletcher, R. B., Baker, J. C., and Harland, R. M. (2006). FGF8 spliceforms mediate early mesoderm and posterior neural tissue formation in Xenopus. Development 133, 1703-1714.

Gammill, L. S., and Bronner-Fraser, M. (2003). Neural crest specification: migrating into genomics. Nat. Rev. Neurosci. 4, 795-805.

Glavic, A., Honore, S. M., Feijoo, C. G., Bastidas, F., Allende, M. L., and Mayor, R. (2004). Role of BMP signaling and the homeoprotein Iroquois in the specification of the cranial placodal field. Dev. Biol. 272, 89-103.

Harland, R. M. (1991). In situ hybridization: an improved whole-mount method for Xenopus embryos. Methods Cell Biol. 36, 685-695.

Hopwood, N. D., Pluck, A., and Gurdon, J. B. (1989). A Xenopus mRNA related to Drosophila twist is expressed in response to induction in the mesoderm and the neural crest. Cell 59, 893-903.

Huang, X., and Saint-Jeannet, J. P. (2004). Induction of the neural crest and the opportunities of life on the edge. Dev. Biol. 275, 1-11.

Jamrich, M., and Sato, S. (1989). Differential gene expression in the anterior neural plate during gastrulation of Xenopus laevis. Development 105, 779-786.

Jonas, E. A., Snape, A. M., and Sargent, T. D. (1989). Transcriptional regulation of a Xenopus embryonic epidermal keratin gene. Development 106, 399-405.

Katagiri, C., Maeda, R., Yamashika, C., Mita, K., Sargent, T. D., and Yasumasu, S. (1997). Molecular cloning of Xenopus hatching enzyme and its specific expression in hatching gland cells. Int. J. Dev. Biol. 41, 19-25.

Kintner, C. R., and Melton, D. A. (1987). Expression of Xenopus N-CAM RNA in ectoderm is an early response to neural induction. Development 99, 311325 .

Knecht, A. K., and Bronner-Fraser, M. (2002). Induction of the neural crest: a multigene process. Nat. Rev. Genet. 3, 453-461.

Kolm, P. J., and Sive, H. L. (1995). Efficient hormone-inducible protein function in Xenopus laevis. Dev. Biol. 171, 267-272.

LaBonne, C., and Bronner-Fraser, M. (1998). Neural crest induction in Xenopus: evidence for a two-signal model. Development 125, 2403-2414.
Lamb, T. M., Knecht, A. K., Smith, W. C., Stachel, S. E., Economides, A. N., Stahl, N., Yancopoulous, G. D., and Harland, R. M. (1993). Neural induction by the secreted polypeptide noggin. Science 262, 713-718.

Le Douarin, N. M. Creuzet, S., Couly, G., and Dupin, E. (2004). Neural crest cell plasticity and its limits. Development 131, 4637-4650.

Litsiou, A., Hanson, S., and Streit, A. (2005). A balance of FGF, BMP and WNT signalling positions the future placode territory in the head. Development 132, 4051-4062

Mayor, R., Morgan, R., and Sargent, M. G. (1995). Induction of the prospective neural crest of Xenopus. Development 121, 767-777.

McGrew, L. L., Takemaru, K., Bates, R., and Moon, R. T. (1999). Direct regulation of the Xenopus engrailed-2 promoter by the Wnt signaling path way, and a molecular screen for Wnt-responsive genes, confirm a role for Wnt signaling during neural patterning in Xenopus. Mech. Dev. 87, 21-32.

Meulemans, D., and Bronner-Fraser, M. (2004). Gene-regulatory interactions in neural crest evolution and development. Dev. Cell 7, 291-299.

Mizuseki, K., Kishi, M., Matsui, M., Nakanishi, S., and Sasai, Y. (1998) Xenopus Zic-related-1 and Sox-2, two factors induced by chordin, have distinct activities in the initiation of neural induction. Development 125, 579-587.

Monsoro-Burq, A. H., Fletcher, R. B., and Harland, R. M. (2003). Neural crest induction by paraxial mesoderm in Xenopus embryos requires FGF signals. Development 130, 3111-3124.

Monsoro-Burq, A. H., Wang, E., and Harland, R. (2005). Msx1 and Pax3 cooperate to mediate FGF8 and WNT signals during Xenopus neural crest induction. Dev. Cell 8, 167-178.

Nieuwkoop, P. D., and Faber, J. (1967). Normal table of Xenopus laevis (Daudin), Amsterdam, The Netherlands: North Holland Publishing Company.

O'Donnell, M., Hong, C. S., Huang, X., Delnicki, R. J., and Saint-Jeannet, J. P. (2006). Functional analysis of Sox8 during neural crest development in Xenopus. Development 133, 3817-3826.

Pandur, P. D., and Moody, S. A. (2000). Xenopus Six1 gene is expressed in neurogenic cranial placodes and maintained in the differentiating lateral lines. Mech. Dev. 96, 253-257.

Saint-Jeannet, J. P., He, X., Varmus, H. E., and Dawid, I. B. (1997). Regulation of dorsal fate in the neuraxis by Wnt-1 and Wnt-3a. Proc. Natl. Acad. Sci. USA 94, 13713-13718.

Sato, T., Sasai, N., and Sasai, Y. (2005). Neural crest determination by coactivation of Pax3 and Zic1 genes in Xenopus ectoderm. Development 132, 2355-2363.

Schambony, A., Hefele, J. A., Gentzel, M., Wilm, M., and Wedlich, D. (2003). A homologue of cysteine-rich secretory proteins induces premature degradation of vitelline envelopes and hatching of Xenopus laevis embryos. Mech. Dev. 120, 937-948

Schlosser, G. (2005). Evolutionary origins of vertebrate placodes: insights from developmental studies and from comparisons with other deuterostomes. J. Exp. Zool. B Mol. Dev. Evol. 304, 347-399.

Seo, H. C., Sætre, B. O., Håvik, B., Ellingsen, S., and Fjose, A. (1998). The zebrafish Pax3 and Pax7 homologues are highly conserved, encode multiple isoforms and show dynamic segment-like expression in the developing brain. Mech. Dev. 70, 49-63.

Smith, W. C., and Harland, R. M. (1992). Expression cloning of noggin, a new dorsalizing factor localized to the Spemann organizer in Xenopus. Cell 70, $829-840$.

Streit, A. (2004). Early development of the cranial sensory nervous system: from a common field to individual placodes. Dev. Biol. 276, 1-15.

Suzuki, A., Ueno, N., and Hemmati-Brivanlou, A. (1997). Xenopus Msx1 mediates epidermal induction and neural inhibition by BMP4. Development 124, 3037-3044

Villanueva, S., Glavic, A., Ruiz, P., and Mayor, R. (2002). Posteriorization by FGF, Wnt, and retinoic acid is required for neural crest induction. Dev. Biol. 241, 289-301.

Wolda, S. L., Moody, C. J., and Moon, R. T. (1993). Overlapping expression of Xwnt-3a and Xwnt-1 in neural tissue of Xenopus laevis embryos. Dev. Biol. 155, $46-57$.

Wu, J., Saint-Jeannet, J. P., and Klein, P. S. (2003). Wnt-frizzled signaling in neural crest formation. Trends Neurosci. 26, 40-45. 